\title{
Discovery of New Mutation Sites of KIF21A Gene in Chinese Patients with Congenital Fibrosis of the Extraocular Muscles
}

\section{Cuiran Xia}

Moorfields Eye Hospital

\section{Ruilan Shi}

Aier Eye Hospital (JinZhong)

\section{Lianghuan Nan}

Moorfields Eye Hospital

Jing Hao

Aier Eye Hospital (JinZhong)

\section{Yading Jia}

Aier Eye Hospital (TaiYuan)

Danjie Li ( 2015 lidanjie@sina.com )

Aier Eye Hospital (ChengDu) https://orcid.org/0000-0001-6957-8263

\section{Research article}

Keywords: Congenital fibrosis of the extraocular muscles; KIF21A gene; Mutation

Posted Date: May 27th, 2020

DOI: https://doi.org/10.21203/rs.3.rs-28924/v1

License: (9) (i) This work is licensed under a Creative Commons Attribution 4.0 International License. Read Full License 


\section{Abstract}

Background Congenital fibrosis of the extraocular muscles (CFEOM) is a rare hereditary nonprogressive disorder characterized by bilateral ptosis, which shows severely limited ocular motility. We reported a new mutation site of KIF21A gene in a Chinese family with congenital fibrosis of the extraocular muscles type 1 (CFEOM1).

Methods A retrospective study of case series was conducted in this study. Standard ocular examinations were performed on 10 family members in a CFEOM1 family. Next-generation sequencing (NGS) was performed to sequence 828 genes related to oculopathy. The splice sites of the KIF21A gene were analyzed to detect the existence of mutations.

Results The five patients with CFEOM1 were found in the family members of a Chinese family on three generations. A new KIF21A pathogenic SNP mutation site KIF21A-ex20 c.2821C > T (p.Arg941Trp) was identified by comparing with human genome reference genes and Sanger sequencing. .

Conclusions A new KIF21A pathogenic SNP mutation site KIF21A-ex20 c.2821C > T (p.Arg941Trp) could possibly be a major disease-causing gene for the Chinese family with CFEOM1.

\section{Background}

Congenital fibrosis of the extraocular muscles (CFEOM) is a rare autosomal dominant hereditary eye disease, which has been reported at a rate of $1 / 230000^{[1]}$. CFEOM's main phenotype is a hereditary ocular motility disorder characterized by congenital ptosis and non-progressive restrictive ophthalmoplegia, which can be accompanied by abnormalities in other systems throughout the body. So far, CFEOM has been classified into 4 types according to genetic and clinical characteristics: CFEOM1, CFEOM2, CFEOM3, and Tukel syndrome. Of which CFEOM1 is the most common type ${ }^{[2,3]}$, and it is mainly caused by mutations in the KIF21A gene R954W ${ }^{[5-7]}$.

This study identified a new heterozygous missense mutation in KIF21A p.Arg941Trp by high-throughput second-generation sequencing and Sanger sequencing in a Chinese family with CFEOM1.

\section{Methods}

In this retrospective study, we reviewed the medical records of patients with CFEOM1 from January 2018 to July 2019 at Aier Eye Hospital (Jinzhong and Taiyuan). Ten subjects in a family of three generations was inquired about the current and previous medical history, family history in details, and they were subjected to comprehensive ophthalmic examinations including best - corrected vision acuity, Slit lamp and ophthalmoscope. The Declaration of Helsinki was strictly followed in this study.

CFEOM1 diagnostic criteria: congenital ptosis and limited eye movement, especially upward rotation restriction. In primary gaze, the eye position is downward. Its upward rotation could not pass the middle 
line and the horizontal movement was severely limited. The two patients of them with CFEOM 1 were tested for genetic tests. The eye chip from Hangzhou Dean Medical Test Center was used to perform Next Generation sequencing (NGS) technology on the target area for gene sequencing of samples.

The eye chip from Hangzhou Dean Medical Test Center was used to perform Next Generation sequencing (NGS) technology on the target area for gene sequencing of samples (two patients with CFEOM1).

The DNA library was first created and it is tested by Agilent 2100 Bioanalyzer and BMG for fragment size and concentration. After passing the test, the library with different data volumes was pooled and quantified. Then, the pooled library was cyclized single-stranded. After the DNA nano balls (DNB) was generated using cyclized library, the high-throughput sequencer MGISEQ-2000 was used to perform continuous bidirectional sequencing and read the raw sequencing data. Detection quality control indicators average coverage is 99.29 (20X), and average sequencing depth is 129.33 .

\section{Results}

\section{1هCLINICAL FINDINGS}

Family and medical histories were carefully reviewed. In one family of family members in three generations, five patients with typical CFEOM1 were identified. (Fig. 1). The pattern of the onset CFEOM1 in this family showed autosomal dominant inheritance. In this family, proband and his mother $\square$ sister $\square$ uncle grandmother suffered from CFEOM1. All patients exhibited severe congenital involvement. Figure 2 showed the appearance of the eyes of the mother and proband ( $₫ 3$ and $\varangle 3$ patients): they shared the typical clinical features of CFEOM1 .These features included bilateral congenital non-progressive ptosis, restricted extraocular muscle paralysis, severely impaired vertical motility with an inability to raise either eye above the horizontal midline and variable impaired horizontal motility, fixation of the eyeballs to the lateral subluxation in primary gaze, chin-up position of the head and absence of Bells phenomenon. Their eyes' upward rotation could not pass the middle line and the horizontal movement was severely limited (Fig. 2). The forced induction test demonstrated marked restriction in passive elevation of the globes in all affected patient.

\section{2ХGENETIC TEST RESULTS}

High-throughput sequencing technology was performed on 828 genes related to hereditary ophthalmopathy in this study. Combined with the clinical symptoms of the patients, one pathogenic mutation, KIF21A-ex20 c.2821C > T (p.Arg941Trp) was detected and heterozygous missense mutated (Fig. 3). ClinVar database included and annotated this mutation as a disease-causing gene. The conserved sequencing of its protein by SIFT and Polyphen-2 software showed that the mutation site was detected in both patients, so it was determined to be a pathogenic mutation. The inheritance of KIF21A gene-related congenital extraocular muscle fibrosis is autosomal dominant inheritance, which is presumed to be the main cause of disease in patients. In this study, genetic analysis documented the 
presence of a heterozygous mutation, c.2821C > T (p.Arg941Trp), in the KIF21A gene within the two patients of the one family. This mutation was not detected in the unaffected family members or in the unrelated control subjects from the same population.

\section{Discussion}

Patients with congenital fibrosis of the extraocular muscles syndrome have non-progressive eye movement disorders, accompanied with various ptosis, eye position fixed on the lateral-downward, and severe bilateral eye movement restrictions. The CFEOM is divided into four types according to its genetic mutations and genetic types, of which CFEOM1 is a rare autosomal dominant genetic disease, and its typical clinical manifestation is congenital non-progressive blepharoptosis, fixation of eyeball at lower index of at least $10^{\circ}$, the upward movement cannot exceed the midline and the horizontal motion is restricted to varying degrees. CFEOM2 is an autosomal recessive inheritance. Patients with CFEOM2 have congenital blepharotosis, partial or complete fixation of the eyeball in the oblique position, and lack of free eye movement. CFEOM3 is an incomplete autosomal dominant inheritance, and its clinical phenotype varies. The forth type is atypical. In our study, all five patients were typical CFEOM1. .

In 2003, Uvama et al. ${ }^{[4]}$ reported that KIF21A gene mutation is closely related to CFEOM1, and think that CFEOM1 was caused by KIF21A encoded kinesin missense mutations. Subsequently, researchers found more than 10 new mutation sites in the KIF21A gene from patients with CFEOM1 families in different nationalities and regions. The most popular one was $2860 \mathrm{C}>\mathrm{G}(\mathrm{R} 954 \mathrm{~W})$ in exon 21 , with a mutation rate of $72 \%$, followed by the $2861 \mathrm{G}>\mathrm{A}$ (R954Q) mutation in exon 21 with a mutation rate of $13 \%$ and a mutation rate of up to $85 \%$. Tiab ${ }^{[5]}$ studied the R954W mutation in all patients by studying three CFEOM1 families from France, Switzerland, and Turkey and one sporadic case from Iran. Chen ${ }^{[7]}$ found two families of CFEOM1 and CFEOM3 with a heterozygous KIF21A c.2860 C > T mutation

In our study, KIF21A-ex20 c.2821C > T (p.Arg941Trp) was detected as a heterozygous missense mutation by studying pedigree. The KIF21A gene was included in the ClinVar database and annotated as a diseasecausing gene. Functional tests showed that the impaired function of the gene resulted CFEOM1 phenotype; and other mutations at the same site of the gene (R941L, R941Q, R954W) were reported in patients with CFEOM ${ }^{[6-8]}$. KIF21A-ex20 c.2821C > T (p.Arg941Trp) found in this study belongs to heterozygous missense mutation, and the frequency of carrying this mutation in East Asian populations has not been reported. The conserved sequencing of its protein by SIFT and Polyphen-2 software showed that the mutation site was detected in both patients, so it was determined to be a pathogenic mutation. The inheritance of KIF21A gene-related congenital extraocular muscle fibrosis is autosomal dominant inheritance, which is presumed to be the main cause of disease in patients. KIF21A-ex20 c.2821C > T (p.Arg941Trp) is detected as a heterozygous missense mutation by studying pedigree, is a new mutation sites of KIF21A gene in Chinese patients with congenital fibrosis of the extraocular muscles.

\section{Conclusion}


We conducted a retrospective study of case series. The five patients with CFEOM1 were found in the ten people of a Chinese family on three generations. We found A new KIF21A pathogenic SNP mutation site

KIF21A-ex20 c.2821C > T (p.Arg941Trp) in two patients of a family with CFEOM1. The mutation is highly possible a major disease-causing gene for the Chinese family with CFEOM1.

\section{Abbreviations}

CFEOM: Congenital fibrosis of the extraocular muscles; NGS: Next Generation sequencing;

\section{Declarations}

Acknowledgements

None.

Funding

None.

\section{Availability of data and materials}

The data sets used and/or analyzed during the current study are available from the corresponding authors (Yading Jia and Cuiran Xia) on reasonable request.

\section{Authors' contributions}

R.X., Y.J., designed the study, and collected data and wrote the manuscript; D.L., designed, wrote and revise the manuscript; R.S., N.L., J.H., collected and analysis patients. All authors made substantial contribution to this manuscript meeting authorship criteria, agreed to be accountable for all aspects of the work and have read and approved the final version.

\section{Ethics approval and consent to participate}

This study followed the tenets of the Declaration of Helsinki and was approved by the Ethics Committee of Aier Eye Hospital (IRB No. J2019-25). Informed written consent was obtained from all participants or their parents.

\section{Consent for publication}

This study was consent for publication from all patients in written format. The proband's parents were consent for publication in written format due to the proband is under 18.

\section{Competing interests}

The authors declare that they have no competing interests. 


\section{Publisher's Note}

Springer Nature remains neutral with regard to jurisdictional claims in published maps and institutional affiliations.

\section{Open Access}

This article is distributed under the terms of the Creative Commons Attribution 4.0 International License (http://creativecommons.org/licenses/by/4.0/), which permits unrestricted use, distribution, and reproduction in any medium, provided you give appropriate credit to the original author(s) and the source, provide a link to the Creative Commons license, and indicate if changes were made. The Creative Commons Public Domain Dedication waiver (http://creativecommons.org/publicdomain/zero/1.0/) applies to the data made available in this article, unless otherwise stated.

\section{References}

\1囚Reck AC, Manners R, Hatchwell E. Phenotypic heterogeneity may occur in congenital fibrosis of the extraocular muscles.Br J Ophthalmol,1998;82:676-679.

ه2 $₫$ Yazdani A MD,Elias I, Traboulsi MD. Classification and surgicalmanagement of patients with familial and sporadic forms of congenitial fibrosis of the extraocular muscles. Ophthamology.2004;111:10351042.

$\varangle 3 \llbracket$ Dan Wang, Longyan Yang, Yue Song. congenital fibrosis of the extraocular muscles $₫ / N T J$ Ophthalmolo. 2005;5(6):1243-1247.

\4囚Uyama E,Yamada K,Kawano H,et al.A Japanese family with FEOM1-linked congenitial type1 associated with spinal canal stenosis and refinement of the FEOM1 critical region[J].Neuro Dis,2003,13(6):472-478.

\5囚Tiab L, d' Alleves Manzi V, Borruat FX, et al. Mutation analysis of KIF21A in congenitial fibrosis of the extraocular muscles (CFEOM) patients[J].Ophthalmic Genet,2004,25.

\6هYamada K, Chan WM, Andrews C, et al. Identification of KIF21 A mutations as a rare cause of congenital fibrosis of the extraocular muscles type 3 (CFEOM3).

Invest Ophthalmol Vis Sci. 2004;45(7):2218-23.

Q7هYamada K, Andrews C, Chan WM, et al. Heterozygous mutation of the kinesin Kif21A in congenital fibrosis of the extrocular muscles type 1 ( CFEOM 1).Nat Genet.2003;35(4):318-21.

\8\ Jingchang Chen, Qingqing Ye, Danming Deng, et al. KIF21A mutation in two Chinese families with congenital fibrosis of the extraocular muscles type 1 and 3 . Molecular Medicine reports.2016(14):31453151. 
Figures

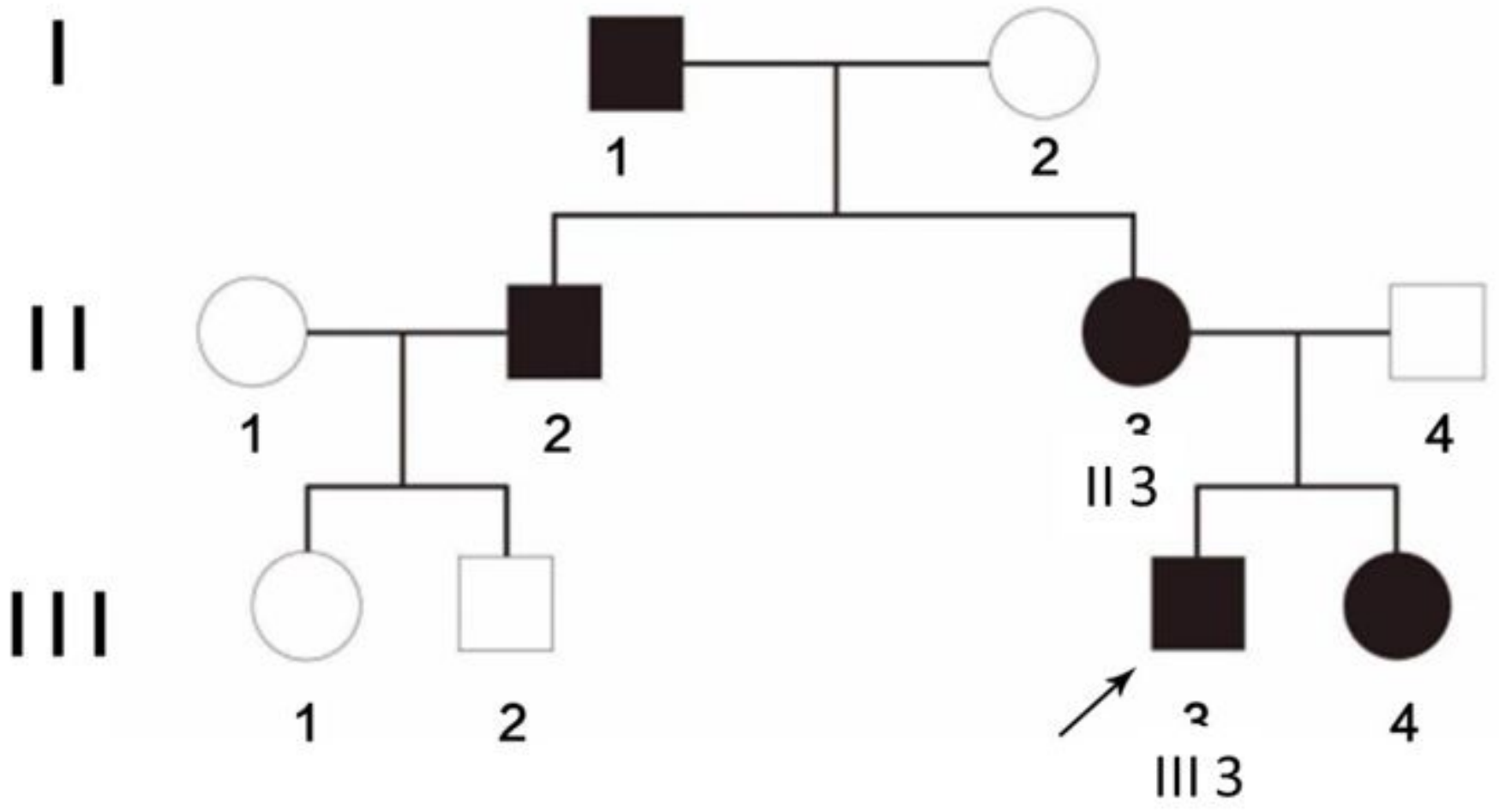

Figure 1

Pedigrees of the family identified with congenital fibrosis of the extraocular muscles. Affected family members are designated by solid symbols. Genetic analysis was performed on individuals with II 3 and III 3 shown below the symbols 

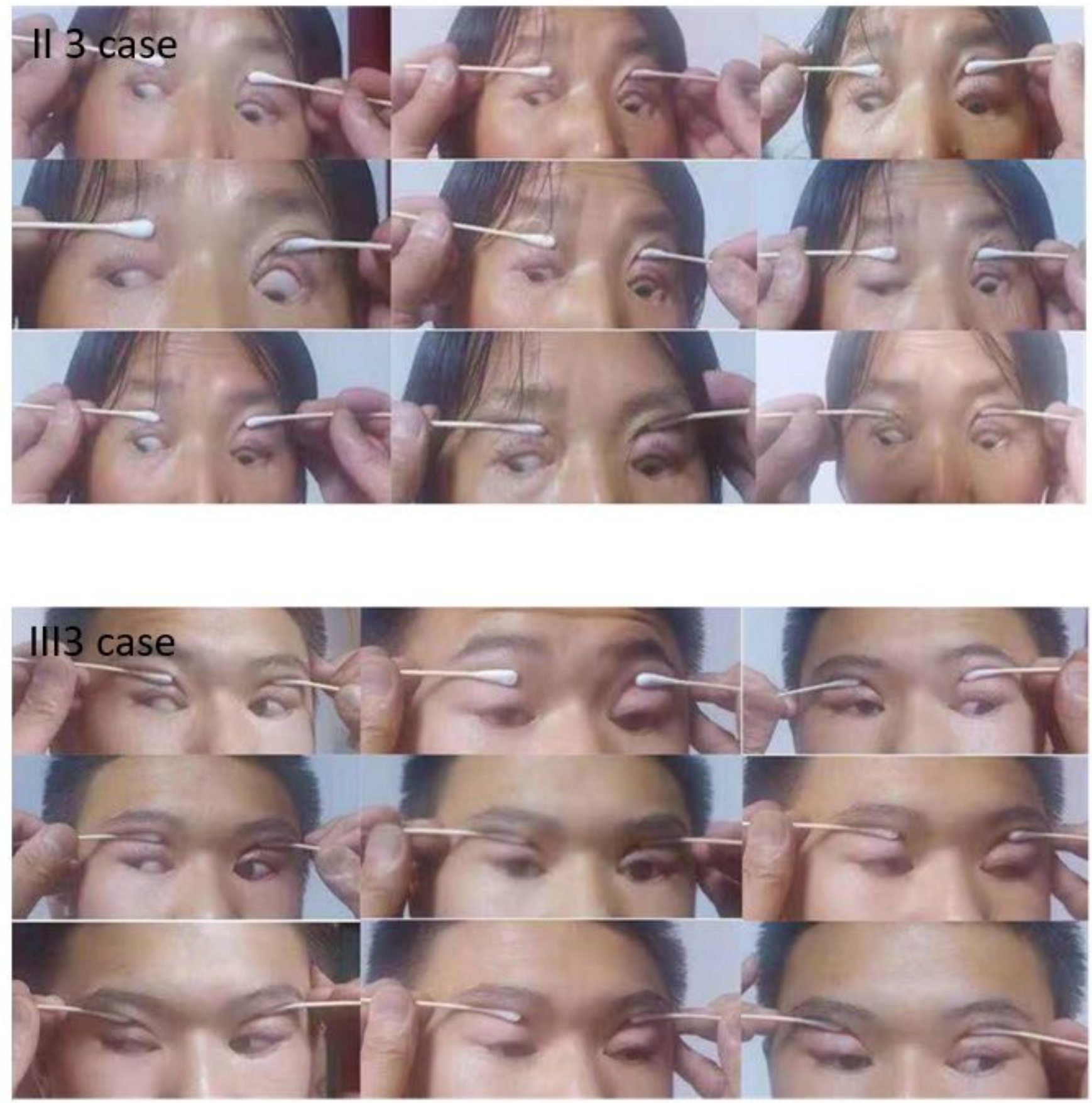

\section{Figure 2}

The appearance of the eyes of the mother (Top, II 3 case) and proband (Bottom, III 3 case): drooping of the upper eyelids of both eyes, restricted extraocular muscle paralysis, fixation of the eyeballs to the lateral subluxation, but no upward movement of the midline, limited horizontal movement, compensating head position, and jaw lifting. 
Reference sequence: AGTTCCTCCCGTTGCTATTGA

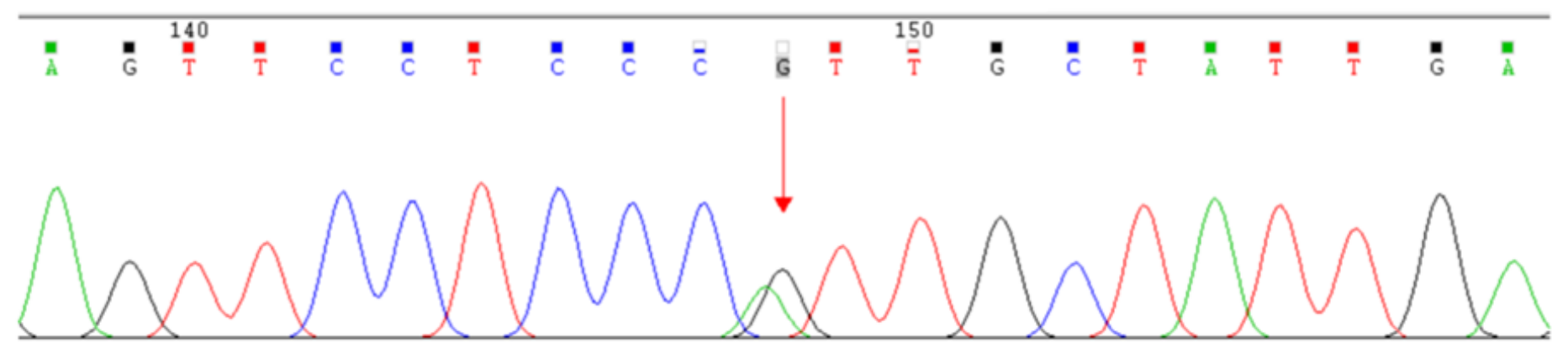

III 3 case: KIF21 A-ex20c.2821C > T (p.Arg941Trp) Sanger sequencing chart (Positive detection)

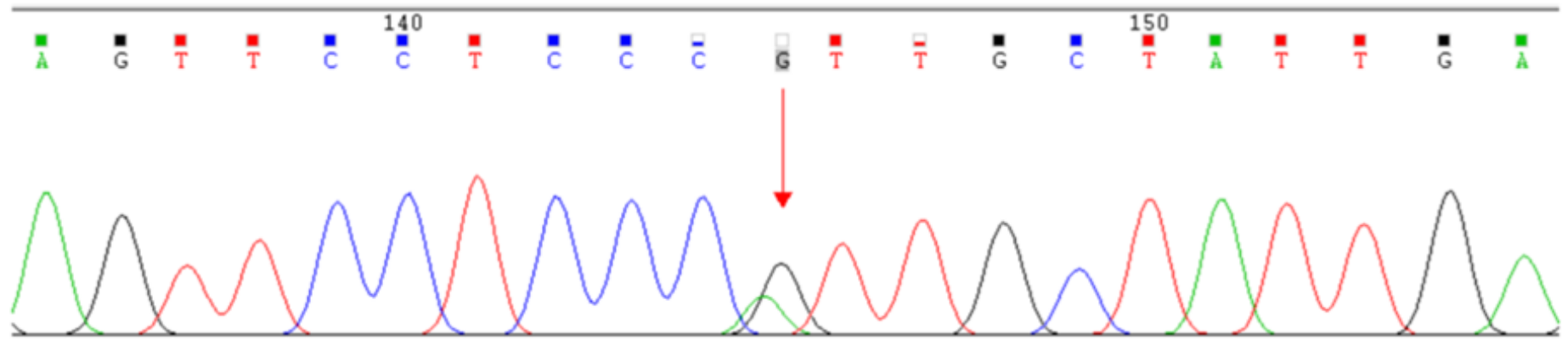

II 3 case: KIF21 A-ex20c.2821C $>$ T (p.Arg941Trp) Sanger sequencing chart (Positive detection)

\section{Figure 3}

Sequencing and mutation sites of exon 21 of the patient's KIF21A gene in the II 3 and III 3 affected members of the family. The position of the mutation is marked by an arrow and the corresponding wild-type sequence is shown as a control. 